\title{
Laparotomy-Assisted Direct Cholangioscopy for Bile Duct Stone Removal via an Incision of the Jejunum
}

\author{
Shinya Sugimoto $^{a}$ Toji Murabayashi $^{a}$ Ayako Ichikawa $^{a}$ Keita Sato $^{\mathrm{b}}$ \\ Akira Kamei $^{a}$ \\ aDepartment of Gastroenterology, Ise Red Cross Hospital, Ise, Japan; bepartment of \\ Surgery, Ise Red Cross Hospital, Ise, Japan
}

\section{Keywords}

Anastomosis · Roux-en-Y · Endoscopic retrograde cholangiopancreatography

\begin{abstract}
A 77-year-old man presented to our hospital with epigastric pain. He had previously undergone hepatic left lateral segmentectomy, cholangiojejunostomy, and Roux-en-Y reconstruction at 42 years of age for intrahepatic stones and liver abscesses. Abdominal computed tomography and magnetic resonance cholangiopancreatography revealed bile duct stones and intrahepatic bile duct dilation of the caudate lobe. Bile duct drainage for the caudate lobe was necessary; however, the volume of his caudate lobe was very small, making percutaneous transhepatic biliary drainage (PTBD) or endoscopic ultrasound-guided biliary drainage (EUSBD) difficult. Therefore, we attempted laparotomy-assisted endoscopic biliary drainage. Under general anesthesia, an incision was made on the jejunum approximately $15 \mathrm{~cm}$ from the Y-leg anastomosis. An esophagogastroduodenoscope was directly inserted into the common hepatic duct anastomosed with the jejunum. The caudate lobe branch had severe stenosis, and the area upstream of the stenosis was filled with stones, sludge, and pus. The biliary stenosis was dilated using a balloon, and the stones were completely removed using a basket and a balloon catheter. There are various methods of biliary and pancreatic surgery and gastrointestinal reconstruction, and there are cases in which PTBD, EUS-BD, and endoscopic retrograde cholangiopancreatography (ERCP) with an enteroscope are difficult. In such cases, ERCP under laparotomy could be a good treatment option.
\end{abstract}


Sugimoto et al.: Laparotomy-Assisted Cholangioscopy

\section{Introduction}

Endoscopic retrograde cholangiopancreatography (ERCP) for the removal of a bile duct stone in patients who had surgically altered anatomy, especially Roux-en-Y (R-Y) anastomosis, is challenging. Besides, in patients who have undergone laparotomy in the past, endoscopic insertion may be difficult due to abdominal firm adhesions. In recent times, balloonassisted enteroscopy and endoscopic ultrasonography are available, which can make bile duct drainage challenging in clinical situation. Balloon-assisted enteroscopy [1-3] and endoscopic ultrasonography [4] have remarkably improved biliary drainage of surgically altered gastrointestinal tracts. While there are various methods of biliary and pancreatic surgery and gastrointestinal reconstruction, there are cases in which percutaneous transhepatic biliary drainage (PTBD), endoscopic ultrasound-guided biliary drainage (EUS-BD), and ERCP with an enteroscope are difficult [5]. ERCP for patients with a surgically altered gastrointestinal tract is still challenging. Herein, we report a case of ERCP using a small-intestinal incision approach for the removal of a common bile duct (CBD) stone that had developed after hepatic left lateral segmentectomy, cholangiojejunostomy, and R-Y reconstruction for liver abscesses.

\section{Case Report/Case Presentation}

A 77-year-old man presented with epigastric pain. He had previously undergone hepatic left lateral segmentectomy, cholangiojejunostomy, and R-Y reconstruction (Fig. 1) at 42 years of age for intrahepatic stones and liver abscesses. His length of stay in the hospital at that time (42 years) was about 3 months, suggesting that it was a very serious infectious disease. We conducted abdominal computed tomography (Fig. 2a) and magnetic resonance cholangiopancreatography (Fig. 2b) which revealed bile duct stones and intrahepatic bile duct dilation of the caudate lobe. He was diagnosed with segmental cholangitis, and antibiotic therapy was initiated. However, on the second day, his epigastric pain worsened, and his body temperature was $41^{\circ} \mathrm{C}$. Bile duct drainage for the caudate lobe was necessary; however, the volume of his caudate lobe was very small, making PTBD or EUS-BD difficult. We attempted biliary drainage using a double-balloon enteroscope, but after 50 min of attempting enteroscopy, we could not reach the Y-leg anastomosis due to postoperative severe adhesions. Immediately after returning to the inpatient room from the endoscopy room, the patient suffered septic shock and was unable to communicate. Hence, we attempted laparotomy-assisted endoscopic biliary drainage. Under general anesthesia, an upper midline abdominal incision was made, and severe adhesions at the Y-limb of the R-Y anastomosis were observed, making it difficult to view the CBD. An incision was made on the slightly oral side of Y-leg anastomosis for insertion of the esophagogastroduodenoscope (GIF-Q260J, Olympus Medical Systems, Tokyo, Japan); however, the endoscope could not reach the CBD for severe adhesion. Hence, we peeled off the postoperative abdominal adhesions as much as possible and made a new incision on the jejunum approximately $15 \mathrm{~cm}$ from Y-leg anastomosis (Fig. 1). The next problem was that there were multiple membranous stenoses in the jejunum that the scope could not pass through (Fig. 2c). We dilated the multiple membranous stenoses by using a balloon (Fig. 2d), and the scope finally reached the liver hilum. The caudate lobe branch was diagnosed with severe stenosis (Fig. 3a), and the area upstream of the stenosis was filled with stones, sludge, and pus (Fig. 3b). The biliary stenosis was dilated using a balloon (Fig. 3c), and the stones were completely removed using a basket and a balloon catheter (Fig. 3d). The patient's postoperative course was uneventful, and he was discharged on postoperative day 10 , with no complications. At 2 months after surgery, he has no recurrence of bile duct stones or postoperative complications.

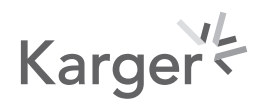




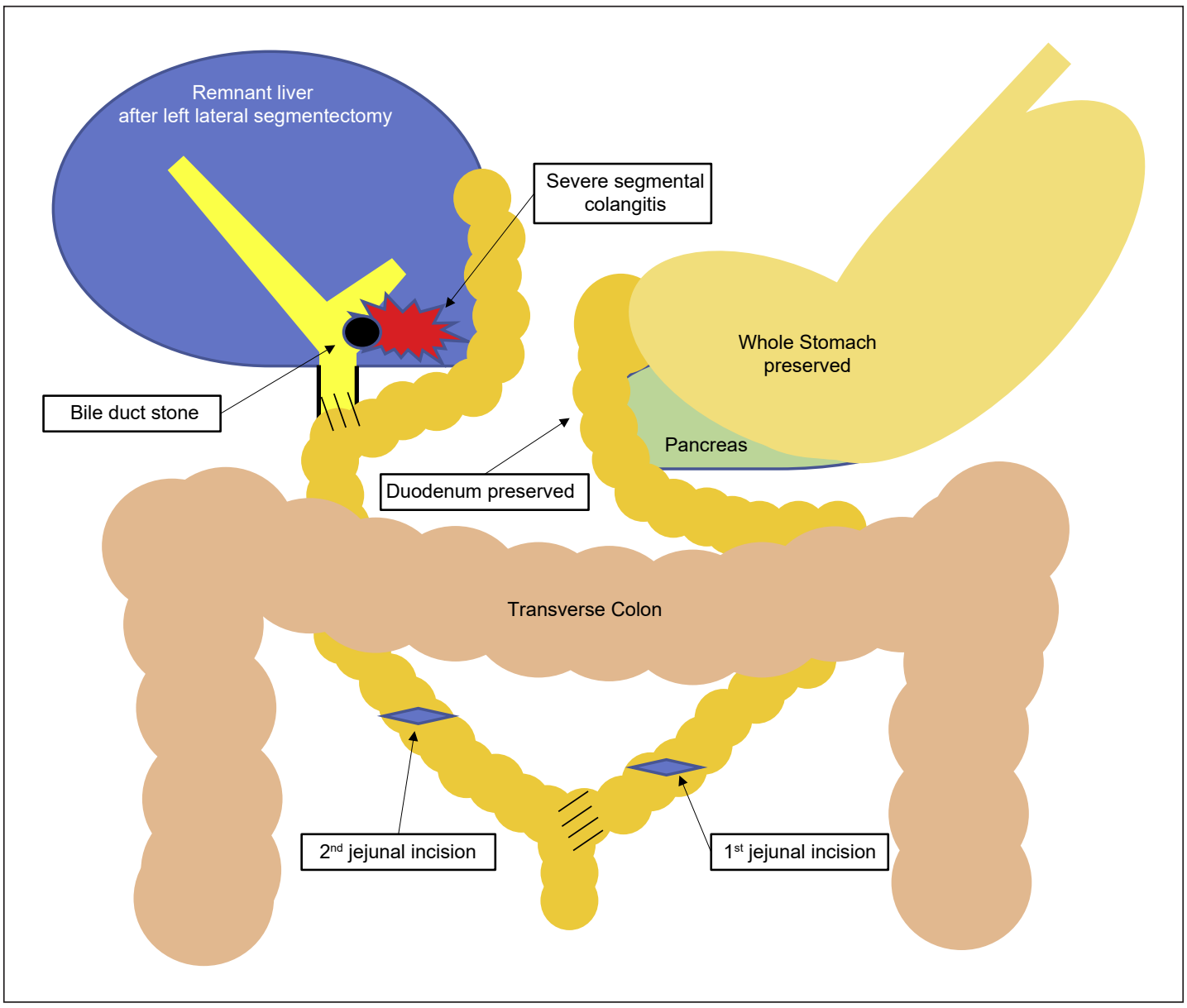

Fig. 1. Schema of the medical condition.

\section{Discussion/Conclusion}

Previous studies have reported the rate of success of reaching the papilla in patients who underwent R-Y anastomosis to be between 71 and $97 \%$, and the ERCP success rate with successful enteroscopy to be $64.1-98 \%$ [1]. The technical success and adverse event rates were 93.8 and $18.8 \%$ in rescue EUS-BD, and 95.0 and $22.5 \%$ in primary EUS-BD, respectively [5].

It is important to note that there are cases in which biliary drainage is not possible with either enteroscope-assisted ERCP or EUS-BD. In the present case, the patient had a history of hepatic left lateral segmentectomy, cholangiojejunostomy, and R-Y reconstruction for intrahepatic stones and liver abscesses. Severe abdominal adhesions were expected. In consultation with the surgeon, it was decided that if enteroscope-assisted ERCP was unsuccessful, we would try open treatment under general anesthesia, instead of transferring the patient to an EUS-BD-capable facility. In fact, the dilated caudate lobe branch was filled with solids (stones, sludge, and pus) that could not be aspirated with a catheter. Even if the drainage tube could be inserted with PTBD or EUS-BD, it would likely not lead to clinical success. Our facility is currently able to perform EUS-BD; however, we felt it was necessary to fully confirm whether the technical success leads to clinical success or not.

To the best of our knowledge, only one previous case has reported clinical success of ERCP with the laparotomic small intestinal incision approach for extraction of a CBD stone 

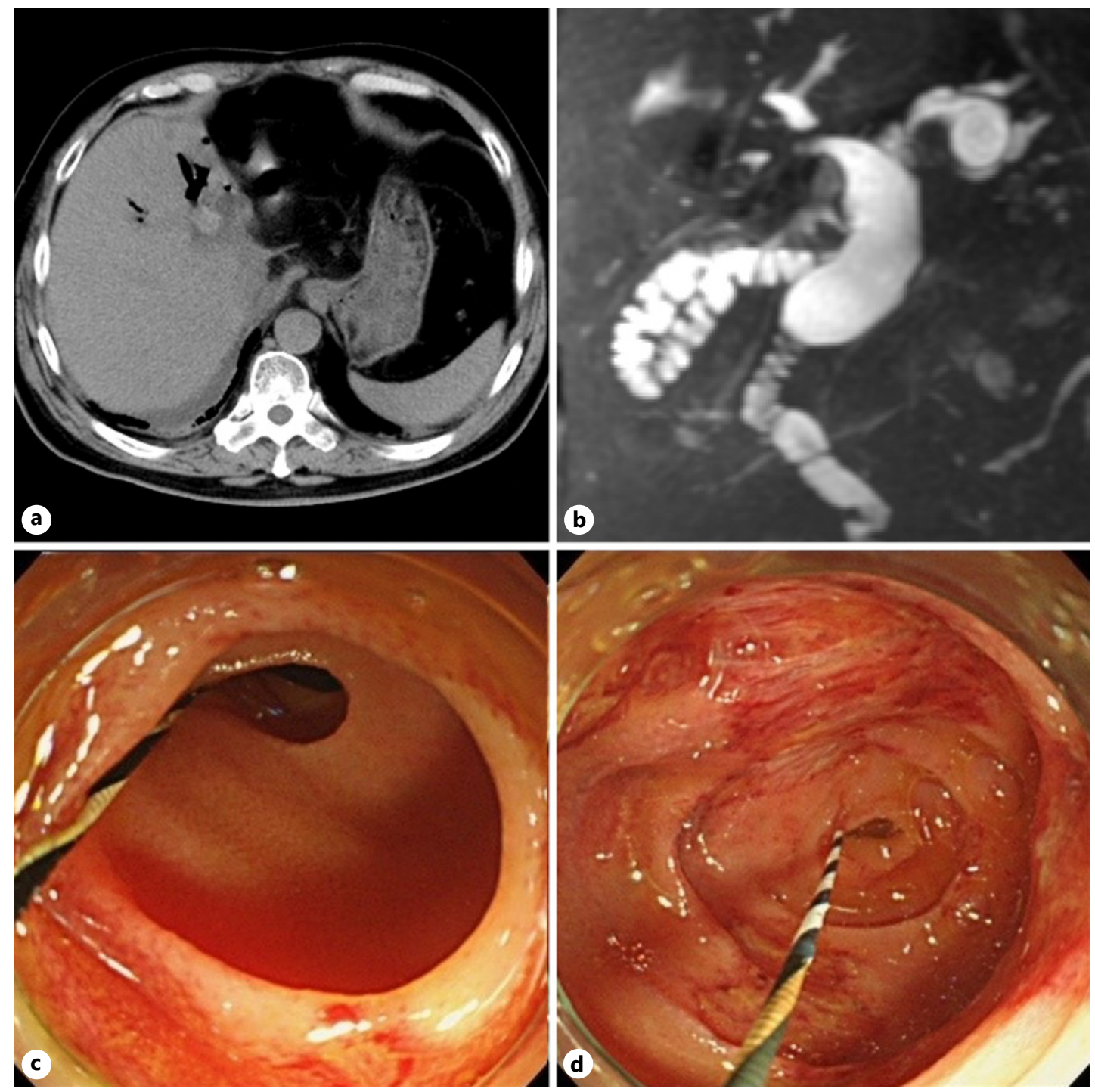

Fig. 2. Preoperative images of the bile duct and biliary drainage. a Computed tomography image of bile duct stones and pneumobilia in the intrahepatic bile duct. b Bile duct stones with intrahepatic bile duct dilation of the caudate lobe imaged with magnetic resonance cholangiopancreatography. c Multiple membranous stenoses in the jejunum. d Dilated multiple membranous stenoses by using a balloon.

following total gastrectomy and R-Y anastomosis [6]. Nakamoto et al. [6] reported that their facility had no available small bowel endoscopes (e.g., single or double balloon) and lacked experience in their use in treating CBD, so they performed a combined endoscopic and open surgery through an incision on the Y-limb of R-Y anastomosis [6]. ERCP for patients with a surgically altered gastrointestinal tract is still challenging, and in such cases, ERCP under laparotomy could be a good treatment option.

\section{Acknowledgements}

This manuscript does not include any nonauthor contributors to acknowledge. 

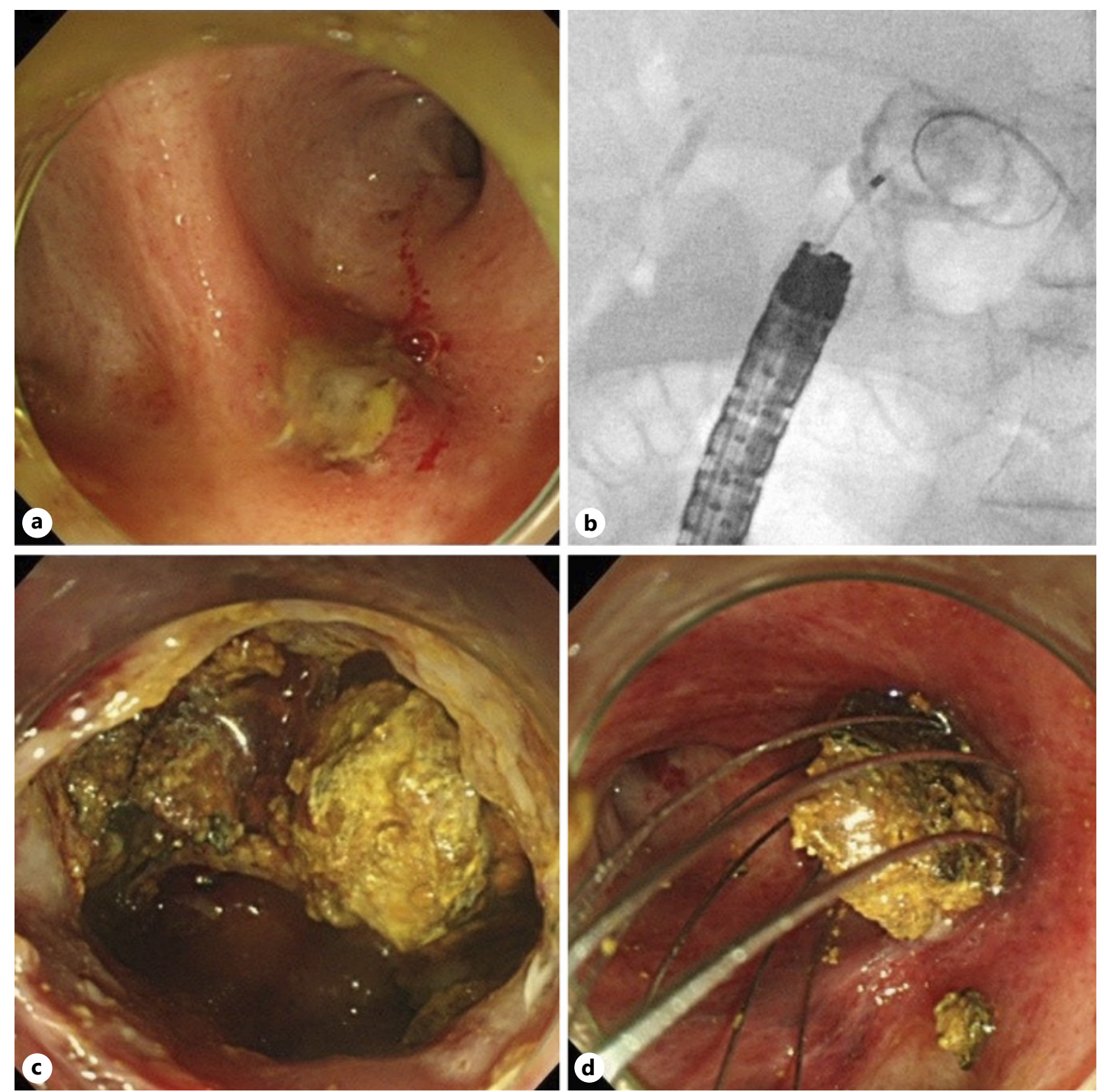

Fig. 3. Intraoperative images and biliary drainage. a The caudate lobe hepatic duct branch showed severe stenosis which originated near the confluence of the left and right hepatic ducts. $\mathbf{b}$ A filling defect in the area upstream of the stenosis of the caudate lobe hepatic duct branch. $\mathbf{c}$ The biliary stenosis was dilated by using a balloon, and the area upstream of the stenosis was filled with stones, sludge, and pus. $\mathbf{d}$ The stones were completely removed using a basket.

\section{Statement of Ethics}

Written informed consent was obtained from the patient for publication of this case report and any accompanying images.

\section{Conflict of Interest Statement}

The authors declare no conflicts of interest. 


\section{Funding Sources}

This manuscript did not receive any funding.

\section{Author Contributions}

Shinya Sugimoto wrote the initial draft of the manuscript. Toji Murabayashi, Ayako Ichikawa, Keita Sato, and Akira Kamei critically reviewed and approved the final version of the manuscript.

\section{References}

1 Katanuma A, Yane K, Osanai M, Maguchi H. Endoscopic retrograde cholangiopancreatography in patients with surgically altered anatomy using balloon-assisted enteroscope. Clin J Gastroenterol. 2014;7(4):283-9.

2 Shao XD, Qi XS, Guo XZ. Endoscopic retrograde cholangiopancreatography with double balloon enteroscope in patients with altered gastrointestinal anatomy: a meta-analysis. Saudi J Gastroenterol. 2017;23(3):150-60

3 Anvari S, Lee Y, Patro N, Soon MS, Doumouras AG, Hong D. Double-balloon enteroscopy for diagnostic and therapeutic ERCP in patients with surgically altered gastrointestinal anatomy: a systematic review and metaanalysis. Surg Endosc. 2021;35(1):18-36.

4 Khashab MA, El Zein MH, Sharzehi K, Marson FP, Haluszka O, Small AJ, et al. EUS-guided biliary drainage or enteroscopy-assisted ERCP in patients with surgical anatomy and biliary obstruction: an international comparative study. Endosc Int Open. 2016;4(12):E1322-7.

5 Nakai Y, Isayama H, Yamamoto N, Matsubara S, Kogure H, Mizuno S, et al. Indications for endoscopic ultrasonography (EUS)-guided biliary intervention: Does EUS always come after failed endoscopic retrograde cholangiopancreatography? Dig Endosc. 2017;29(2):218-25.

6 Nakamoto H, Nishikawa M, Ishikawa T, Yokoyama R, Taketomi A. Endoscopic retrograde cholangiopancreatography (ERCP) with laparotomic small-intestinal incision approach for extraction of a common bile duct (CBD) stone following total gatrectomy and roux-En-Y (R-Y) anastomosis: a case report. Am J Case Rep. 2018 Oct 3;19:1175-8. 